\title{
Investing on the CAPM Pricing Error
}

\author{
José Carlos de Souza Santos, Elias Cavalcante Filho \\ Economics Department, Universidade de São Paulo, São Paulo, Brazil \\ Email: jcdssan@usp.br, e.cavalcante@usp.br
}

How to cite this paper: de Souza Santos, J.C. and Filho, E.C. (2017) Investing on the CAPM Pricing Error. Technology and Investment, 8, 67-82.

https://doi.org/10.4236/ti.2017.81006

Received: January 4, 2017

Accepted: February 19, 2017

Published: February 22, 2017

Copyright $(9) 2017$ by authors and Scientific Research Publishing Inc. This work is licensed under the Creative Commons Attribution International License (CC BY 4.0).

http://creativecommons.org/licenses/by/4.0/

\begin{abstract}
We tested an investment strategy based on the pricing error of the CAPM model. Starting with the Markowitz (1952) [1] methodology, we replaced the standard expected returns vector with the expected errors vector from the CAPM model, assuming that such errors are nonzero and persist over time. When evaluated over the entire examined period, all of the resulting portfolios outperformed the market portfolio. Except for some shorter periods, our hypothesis was fully confirmed. That is, the performance of our alpha portfolios was significantly better than the market portfolio. In other words, the pricing error of the CAPM model seems to be nonzero and to have an inertial component.
\end{abstract}

\section{Keywords}

CAPM, Portfolio Optimization, EWMA, Sharpe Ratio, Certainty Equivalence

\section{Introduction}

The goal of this study is to determine whether the pricing error of the CAPM model by William Sharpe (1964) [2] and Lintner (1965) [3] exhibits an inertial component. Fama and French (2004) [4] state that CAPM is still widely used for pricing in both academia and industry. There is, however, a vast literature, including Banz (1981) [5], Rosenberg, Reid and Lanstein (1985) [6], Fama and French (1993) [7], Jegadeesh and Titman (1993) [8] and Fama and French (2014) [9], which shows that the model's asset pricing is flawed.

The CAPM model states that the expected return of a given asset satisfies the following formula:

$$
E\left(R_{i}-R_{f}\right)=\alpha_{i}+\beta_{i} E\left(R_{m}-R_{f}\right)
$$

where $R_{i}$ is the return on asset $i ; R_{f}$ is the risk-free return; $R_{m}$ is the market return; and $\alpha_{i}$ is the expected pricing error of asset $i$.

Note, however, that the model also assumes that the pricing error is zero, that is, that $\alpha_{i}=0, \forall i \in\{1,2, \cdots, N\}$, where $N$ is the number of available assets. 
This paper, in contrast, assumes that the error is non-zero, and tests whether this pricing error is persistent and may be profitably traded on. Specifically, we test whether excess returns may be obtained by taking long and short positions in assets according to the proportions given by the model's beta parameters, so that the portfolio returns reflect the model pricing error.

Our motivation is as follows. Both the financial market as a whole and individual investors generally assess the performance of a stock portfolio simply by comparing the portfolio return to that of a stock index. The portfolio is therefore evaluated solely by its market risk, disregarding other risk factors such as those specified in Fama and French (1993) [7].

We constructed optimal portfolios using the Markowitz (1952) [1] methodology, but replacing the expected returns vector and the variance and covariance matrices with the expected pricing error and its respective variance and covariance matrices. We then compared the return of the resulting port- folios with the return of the market portfolio.

The result was positive insofar as the constructed ("alpha") portfolios yielded greater-than-market returns when considered over the entire period of available data. On the other hand, over some shorter periods, the performance of the alpha portfolios, as measured via Sharpe ratios and certain equivalents, did not significantly improve on that of the market portfolio.

\section{Literature Review}

The core of Modern Portfolio Theory is based on the work of Markowitz (1952) [1], which earned him the 1990 Nobel Prize in Economics. This core corresponds to the set of Mean-Variance (MV) type models.

The MV models analyse the behavior of a risk-averse investor over a finite time horizon. Starting from a given initial allocation the investor chooses a portfolio by selecting assets and their respective quantities from a set of $\mathrm{N}$ distinct available assets. This decision is made based on the investor's knowledge of 1) the expected returns and 2) the variances and covariances of the available assets. The investor builds the portfolio so as to maximize expected returns for a given level of risk.

While the basic insight of the MV models is relatively simple, their implementation presents two serious problems. First, as reported by De Miguel, Garlappi and Uppal (2007) [10], it is difficult to obtain accurate estimates of the expected returns vector as well as of the variance and covariance matrices of the optimal portfolios.

Second, the literature uses the capital asset pricing model (CAPM) developed by William Sharpe (1964) [2] and John Lintner (1965) [3] to explain the expected asset returns. CAPM relies on the assumption that there is a linear relationship between the market factor, represented by the excess return of the market portfolio, and the sensitivity of the asset to that market factor, known as the asset beta.

However, while the CAPM model is widely used both in academia and in in- 
dustry (see Fama and French (2004) [4]), several studies show that it substantially fails to explain asset returns. Specifically, CAPM consistently yields nonzero pricing errors. The aforementioned studies include for instance 1) Fama and French (1993) [7], who report that the CAPM is unable to explain some returns which are related to the book-to-market ratio and the market value (they moreover propose a new, multifactor CAPM model to fix this problem); and 2) Jegadeesh and Titman (1993) [8], who show that asset returns are positively related to past performance and therefore cannot be explained solely by the asset beta and the market premium.

Hence the CAPM, despite its widespread use, displays some serious empirical failures. It therefore might be possible to build portfolios whose positions take advantage of the model's pricing failures. Specifically, we investigate whether it is possible to earn excess returns by applying the methodology proposed by Markowitz (1952) [1], but replacing the expected returns vector with the CAPM's expected pricing error vector.

\section{Data}

In this paper we used BOVESPA (Bolsa de Valores de São Paulo-Brazil) stock prices, national Brazilian risk-free interest rates, and a series of theoretical portfolio returns corresponding to actual market behavior during the 02/01/2001 to $02 / 01 / 2015$ period. Both the market return history and the risk-free rate were obtained from NEFIN ${ }^{1}$, while the asset prices were collected from Economatica ${ }^{2}$.

The market return was defined as the excess return (i.e., return minus the risk-free rate) of a theoretical market portfolio developed by NEFIN. The risk free rate was given by the 30-day DI swap rate. Assets had to meet the following criteria to be included in the market portfolio:

a) Be the most traded asset of the company, that is, the one with the highest trading volume during the previous year;

b) Have been traded during at least $80 \%$ of the days of the previous year, with a mean financial volume greater than $\mathrm{R} \$ 500,000.00$ per day. If the asset was first listed in the previous year, the period begins on the listing day and ends on the last day of the year;

c) The asset must have been listed before December of the previous year;

The asset price history used the same criteria applied in the selection of the assets in the market portfolio, that is, on a given day the return of an asset was used only if on that day the asset belonged to the market portfolio. Moreover, if an asset was not traded in a given day then we assumed that its price remained constant at the last observed price.

\section{Methodology}

In this section we present the methodology applied in this work. First, we pre${ }^{1}$ Brazilian Center for Research in Financial Economics of the University of São Paulo (Núcleo de Pesquisas em Economia Financeira, www.nefin.com.br).

${ }^{2} \mathrm{~A}$ platform which provides information about Latin America's stock markets, government bonds, the fund industry and various indicators (https://economatica.com/). 
sent how the portfolios were build. After that we show the performance measure used to compare the alphas portfolios with the benchmark used, the market return.

\subsection{Method for Alpha Portfolio Construction}

In order to build the alpha portfolios, we follow some steps. First, we select a time window, then, in this time window, we estimate the CAPM error for each asset in each period of time. Following, we forecast the expected alpha for the next period and we use this expected error as the expected return in a Markowitz portfolio framework.

We apply some constrains on the portfolios weights, as consequence we have different sorts of portfolios, each one defined under different weights constraints. We also apply a stop mechanism in order to limit losses. In the next subsections we explained in detail how we perform the alphas estimation, the alpha forecast and how we define the portfolios weights.

\subsubsection{Alpha Vector and Variance and Covariance Alpha Matrices}

The main working hypothesis of this study is that the CAPM model exhibits inertial pricing errors. That is, we assume that if the model underprices (overprices) an asset during a given period, then it tends to do likewise during the subsequent period. Given this assumption, we shall check whether it is possible to exploit this behavior to earn excess returns.

Investors can take advantage of the pricing error of a given asset to the extent that this error is predictable. If the model is undervaluing the asset and the investor is aware of this, she should take a long position in the asset and simultaneously short a market position whose weight corresponds to the systemic risk of the asset, that is, to the $\beta$ of the CAPM. We call this strategy investing in the assets alpha.

Based on this strategy this paper studies portfolios that were constructed using not the calculated expected return and risk, but instead the expected forecast error and its corresponding variance and covariance matrices.

In this section we explain first the estimation of the asset error and then the estimation of the variance and covariance matrices.

\subsubsection{Alpha Vectors}

To obtain the alpha estimated vector for a specific day $h$ it was necessary to estimate the CAPM model for each asset given the information available at that time, so as to yield the expected estimation error of the model.

Consider the CAPM model

$$
E_{t}\left(R_{i}^{e}\right)=\alpha_{i \mid t}+\beta_{i \mid t} E_{t}\left(R_{m}-R_{f}\right)=\alpha_{i \mid t}+\beta_{i \mid t} E_{t}\left(R_{m}^{e}\right)
$$

with

$$
\begin{gathered}
\beta_{i \mid t} \equiv E_{t}\left(\beta_{i}\right)=\frac{\operatorname{Cov}_{t}\left(R_{i}^{e}, R_{m}^{e}\right)}{\operatorname{Var}_{t}\left(R_{m}^{e}\right)} \\
\alpha_{i \mid t} \equiv E_{t}\left(\alpha_{i}\right)=E_{t}\left(R_{i}^{e}\right)-\hat{\beta}_{i \mid t} E_{t}\left(R_{m}^{e}\right)=E_{t}\left(R_{i}^{e}-\hat{\beta}_{i \mid t} R_{m}^{e}\right)
\end{gathered}
$$


where $R_{i}^{e}$ is the excess return of asset $i ; \alpha_{i \mid t}$ is the expected pricing error of asset $i$ conditional on the information available in period $t ; \beta_{i \mid t}$ is the systemic risk of asset $i$ conditional on period $t ; R_{m}$ is the market return; and $R_{f}$ is the risk-free return.

Note that in order to estimate the expected pricing error $\alpha_{i \mid t}$ of the CAPM model we must first compute each of its realized past values. Below we first summarize the process involved, and later detail its steps.

We perform the following procedure to estimate the expected forecast error for a subsequent period. Given each asset $i \in\{1,2, \cdots, N\}$ and each period $h \in\{T+1, T+2, \cdots\}$, where $N$ is the number of available assets; $H$ the set of days with available data; and $T$ the number of days used in the model estimation:

a) Set the value of $T$, the number of days used in the model estimation;

b) Estimate $\hat{\beta}_{i \mid h}$;

c) Calculate $\hat{\alpha}_{t, i \mid h}=R_{t, i}^{e}-\hat{\beta}_{i \mid h} R_{t, m}^{e}, \forall t \in(h, h-1, h-2, \cdots, h-T+1)$, i.e., calculate each model error in the estimation sample;

d) Given the sequence $\left(\hat{\alpha}_{h,|,|}, \hat{\alpha}_{h-1, i \mid h}, \cdots, \hat{\alpha}_{h-T+1, i \mid h}\right)$, which was estimated in the previous step, estimate $\hat{\alpha}_{i \mid h}$, the expected value of $\hat{\alpha}_{i \mid h}$;

We now explain the details involved in the calculations above.

\subsubsection{Calculating $\beta$}

To obtain $\hat{\beta}_{i \mid h}$ first define

$$
\Sigma_{t, i \mid h}=\left[\begin{array}{c}
R_{t, i}^{e}-\bar{R}_{i \mid h}^{e} \\
R_{t, m}^{e}-\bar{R}_{m \mid h}^{e}
\end{array}\right]\left[\begin{array}{ll}
R_{t, i}^{e}-\bar{R}_{i \mid h}^{e} & R_{t, m}^{e}-\bar{R}_{m \mid h}^{e}
\end{array}\right]
$$

where $\bar{R}_{i \mid t}^{e}$ is the sample mean for asset $i$ during the estimation period, which covers from $(h-T+1)$ to $h$. Therefore $\Sigma_{t, i \mid h}$ is the cross product of the deviation from the mean of the excess return of asset $i$ at time $t$.

Hence

$$
\Sigma_{i \mid h} \equiv E_{h}\left(\Sigma_{t, i \mid h}\right)=\operatorname{Var}_{h}\left(\begin{array}{l}
R_{t, i}^{e} \\
R_{t, m}^{e}
\end{array}\right)=\left[\begin{array}{cc}
\operatorname{Var}_{h}\left(R_{t, i}^{e}\right) & \cdot \\
\operatorname{Cov}_{h}\left(R_{t, i}^{e}, R_{t, m}^{e}\right) & \operatorname{Var}_{h}\left(R_{t, m}^{e}\right)
\end{array}\right], \forall t \leq h
$$

so that $\Sigma_{i \mid h}$ is the variance-covariance matrix conditional on $h$ of the excess returns vector of asset $i$.

We now estimate $\Sigma_{i \mid h}$ assuming that its value may be obtained via the EWMA (exponentially weighted moving average) of the sequence

$$
\begin{aligned}
& \left(\Sigma_{h-T, i \mid h}, \Sigma_{h-T+1, i \mid h}, \cdots, \Sigma_{h, i \mid h}\right): \\
& \hat{\Sigma}_{i \mid h}=\delta \Sigma_{h, i \mid h}+\delta(1-\delta) \Sigma_{h-1, i \mid h}+\delta(1-\delta)^{2} \Sigma_{h-2, i \mid h}+\cdots+\delta(1-\delta)^{\mathrm{T}} \Sigma_{h-T, i \mid h}
\end{aligned}
$$

where $\hat{\Sigma}_{i \mid h}$ estimates $\Sigma_{i \mid h}$ and $\delta$ was fixed at 0.96 .

We finally have that

$$
\hat{\beta}_{i \mid h}=\frac{\widehat{\operatorname{Cov}}_{h}\left(R_{i}^{e}, R_{m}^{e}\right)}{\widehat{\operatorname{Var}}_{h}\left(R_{m}^{e}\right)}
$$




\subsubsection{Calculating $a$}

Given $\hat{\beta}_{i \mid h}, \forall i \in\{1,2, \cdots, N\}$ and $h \in\{T+1, T+2, \cdots, H\}$ we can obtain

$$
\begin{aligned}
& \alpha_{t, i \mid h}=R_{t, i}^{e}-\hat{\beta}_{i \mid h} R_{t, m}^{e}, \\
& \forall i \in\{1,2, \cdots, N\}, h \in\{T+1, T+2, \cdots, H\} \text { and } \\
& t \in\{h, h-1, \cdots, h-T+1\}
\end{aligned}
$$

Analogously to what was done in the estimate of $\Sigma_{i \mid h}$ we use EWMA to estimate $\hat{\alpha}_{i \mid h}$ as follows:

$$
\hat{\alpha}_{i \mid h}=\lambda \hat{\alpha}_{h, i \mid h}+\lambda(1-\lambda) \hat{\alpha}_{h-1, i \mid h}+\lambda(1-\lambda)^{2} \hat{\alpha}_{h-2, i \mid h}+\cdots+\lambda(1-\lambda)^{\mathrm{T}} \hat{\alpha}_{h-T, i \mid h}
$$

where $\hat{\alpha}_{i \mid h}$ estimates $\alpha_{i \mid h}$ and the value of $\lambda$ was chosen to minimize the estimate's mean quadratic error.

\subsubsection{Alpha Variance and Covariance Matrices}

We now explain the steps involved in estimating the variance and covariance matrices of the alpha assets at each moment in time.

We intend to estimate:

$$
\Omega_{h}=E_{h}\left[\begin{array}{ccc}
\operatorname{Var}\left(\alpha_{1}\right) & \ldots & \operatorname{Cov}\left(\alpha_{1} \alpha_{N}\right) \\
\vdots & \ddots & \vdots \\
\operatorname{Cov}\left(\alpha_{N} \alpha_{1}\right) & \ldots & \operatorname{Var}\left(\alpha_{N}\right)
\end{array}\right]
$$

Using the values of $\hat{\alpha}_{i t \mid h}$ which were estimated in the previous section we have that:

$$
\begin{aligned}
& \hat{\Omega}_{t \mid h}=\left[\begin{array}{c}
\hat{\alpha}_{1 \mid t h}-\overline{\hat{\alpha}}_{1 \mid h} \\
\vdots \\
\hat{\alpha}_{N \mid h}-\overline{\hat{\alpha}}_{N \mid h}
\end{array}\right)\left(\begin{array}{lll}
\hat{\alpha}_{1 t \mid h}-\overline{\hat{\alpha}}_{1 \mid h} & \ldots & \left.\hat{\alpha}_{N \mid h}-\overline{\hat{\alpha}}_{N \mid h}\right)
\end{array}\right], \\
& \forall t \in\{h-T, \cdots, h\}
\end{aligned}
$$

where $\overline{\hat{\alpha}}_{\mid h}$ is the sample mean of $\hat{\alpha}_{t h}, t \in\{h, h-1, \cdots, h-T+1\}$.

Now, as in the previous section, we employ EWMA to obtain the following estimate:

$$
\hat{\Omega}_{h}=\delta \Omega_{h \mid h}+\delta(1-\delta) \Omega_{h-1 \mid h}+\delta(1-\delta)^{2} \Omega_{h-2 \mid h}+\cdots+\delta(1-\delta)^{\mathrm{T}} \Omega_{h-T \mid h}
$$

where $\delta=0.96$ is the same decay exponent which we used to estimate $\hat{\Sigma}_{i \mid h}$.

\subsubsection{Portfolios}

Let us now explain the rules used to build the portfolios. We employed three methods to assemble the portfolios: the first one, called Naïve, is the simplest of the three; the other two, which we call Markowitz and Long-only Markowitz, are based on the Markowitz (1952) [1] methodology of optimal portfolios. The latter two differ from each other in terms of the restrictions imposed on each of them.

For each moment $h \in\{T+1, T+2, \cdots, H\}$ we computed the portfolios of all three methodologies using the values obtained as described in section 4.1.

\subsubsection{The Naïve portfolios}

The weigths of each assets alpha in the Naive portfolios are based solely on the 
signs of the expected alphas. The procedure involves three steps: 1) the assets were classified according to the signs of their expected alphas; 2) assets with positive alphas were assigned a portfolio weight of $2 / N_{(+)}$, where $N_{(+)}$is the total number of assets with positive alphas; and 3) assets with negative alphas were assigned a portfolio weight of $-2 / N_{(-)}$, where $N_{(-)}$is the total number of assets with negative alphas. This thereby produced portfolios with (equallyweighted) long positions in assets with positive alphas and (equally-weighted) short positions in assets with negative alphas in every period.

\subsubsection{The Markowitz Portfolios}

The Markowitz portfolios were designed to maximize the expected return conditional on a specific level of risk. The risk level metric used was the standard deviation of the market portfolio observed in the last 252 days relative to the reference date $\left(\sigma_{\text {market } h}\right)$.

The Markowitz portfolios were therefore obtained by solving the following optimization problem:

$$
\begin{array}{ll}
\max _{w_{t}} & w_{t} \alpha_{h \mid h} \\
\text { s.t. } & \\
& w_{t}^{\prime} l=1 ; \\
& w_{t}^{\prime} \Omega_{t} w_{t} \leq \sigma_{\text {market } h} ; \\
& w_{\min } \leq w_{t, i} \leq w_{\max } ;
\end{array}
$$

where $w_{t}$ is the $(1 \times N)$ weight vector; $\boldsymbol{t}$ is an $(N \times 1)$ vector consisting of $1 \mathrm{~s}$; $w_{\min }$ is the minimum acceptable value of $w_{t} ; w_{\max }$ is the maximum acceptable value of $w_{t} ; \alpha_{h \mid h}$ is the $(N \times 1)$ expected alphas vector computed previously; and $\Omega_{t}$ is the $(N \times N)$ alpha variance and covariance matrix.

\subsubsection{The Long-Only Markowitz Portfolios}

The long-only Markowitz portfolios differ from the plain Markowitz portfolios in that the former have an additional restriction on the portfolio weights: every weight must be positive, so that there are no short positions in individual assets.

In the long-only case the weights therefore follow from the solution of the following optimization problem:

$$
\begin{array}{ll}
\max _{w_{t}} & w_{t} \alpha_{h \mid h} \\
\text { s.t. } & \\
& w_{t}^{\prime} l=1 ; \\
& w_{t}^{\prime} \Omega_{t} w_{t} \leq \sigma_{\text {market } \mid h} ; \\
& w_{t, i} \geq 0
\end{array}
$$

\subsubsection{Bounds on Leverage}

The short positions allowed by the Markowitz portfolios imply the possibility of leverage, in the sense that the sum of the weights of long positions of the portfolio may exceed $100 \%$.

We impose a lower bound $w_{\min }$ on the weights to limit the leverage of the portfolio. The goal was therefore to find a value for $w_{\min }$ that solved the fol- 
lowing problem:

$$
\begin{aligned}
& \max _{w_{\min }} \sum_{i=1}^{N}\left|w_{i}\right| \\
& \text { s.t. } \\
& \qquad \sum_{i=1}^{N}\left|w_{i}\right| \leq L ; \sum_{i=1}^{N} w_{i}=1 ; \quad w_{t, i} \leq w_{\max } ;
\end{aligned}
$$

where $L$ is the maximum leverage. Here we use the sum operator (over all assets) instead of the vector $\boldsymbol{t}$ to highlight that the total number of available assets affects the solution.

We illustrate the issue with an example. Assume that we have 12 available assets and that $w_{\max }=2$ and $L=10$. If $w_{\min }=-1$ then the maximum leverage vector $\left(w_{L^{\max }}\right)^{3}$ would be given by:

$$
w_{L^{\max }}=(-1,-1,-1,-1,-1,-1,-1,-1,+1,+2,+2,+2,+2)
$$

Note that all of the restrictions are satisfied except for the leverage bound, since the sum of the absolute values of the weights is equal to 13. This implies that the value of $w_{\min }$ must be set higher: in this example it must be equal to -0.5 , as explained below.

$$
w_{L^{\max }}=(-0.5,-0.5,-0.5,-0.5,-0.5,-0.5,-0.5,-0.5,-0.5,+1.5,+2,+2)
$$

We use the following numerical procedure to find the required value of $w_{\min }$. Given values for $w_{\max }, N$, and $L$ we obtain the vector of maximum leverage for a trial $w_{\min }$ by repeatedly (1) checking whether the restrictions were satisfied and then (2) changing the values of $w_{\min }$ until all of the restrictions were satisfied.

In this study we chose to specify a reasonable value for $\mathrm{L}$ and at the same time pick a value for $w_{\max }$ that is high enough that it does not constitute an active restriction for the solution of the problem.

\subsubsection{Stoploss}

We used a stoploss mechanism: the portfolio position was set to zero whenever the position accumulated a loss greater than or equal to $25 \%$.

\subsubsection{Alpha Portfolios}

By following the previous steps, we have so far assembled a portfolio for each day of the period, except for the initial T early days, which were used only for the initial estimates. Thus, one can consolidate the 21 portfolios into a final portfolio as follows:

a) using the weights obtained with the portfolio assembled with the information available at the time $T$, we invest on date $T+1$;

b) on date $T+2$ we accumulate the return obtained in the previous step and assemble a new portfolio using the information available on $T+1$;

d) we continue the procedure until we obtain 21 portfolios;

e) on date $T+21+1$ we set to zero the position of the portfolio that we have obtained during the previous 21 days and invest its cumulative value on a port- 
folio assembled using the information available up to date $T+21+2$;

f) we continue the procedure by every day discarding a portfolio and replacing it with a new one, so that we are always investing in a combination of 21 portfolios.

The end result is a portfolio consisting always of several portfolios with distinct maturities. The first portfolio was assembled using only the initial information and is invested in for only one day, while the oldest portfolio belongs to the portfolio beginning 21 days ago and will be discarded the following day. The performance of the alpha portfolio is given by the average performance of the 21 portfolios that constitute it. We choose to analyse the portfolios in this way, so our results were less sensitive to the data that the analysis begins.

\subsubsection{Constant Weights or Quantities}

We deal with various types of portfolios. Each alpha is a portfolio consisting of the asset and the market, which becomes more complex as we add more and more alpha assets. These in turn become even more elaborate when we consolidate portfolios of distinct maturities into a single portfolio.

As we accumulate the portfolio returns, this process raises the following question: what should be kept constant over time, the asset weights or the asset quantities?

We considered both approaches. We first kept weights constant-we call this the Equal Weight approach. Regardless of past performance all weights were kept fixed and equal to the originally calculated weights. This involves of course selling the outperforming assets and buying the underperforming assets so that so that they remain constant as portions of the whole portfolio.

In the second approach, called Value Weight, the asset weights vary depending on their past performance. Outperforming assets gain weight and underperforming assets lose weight in the portfolio. This is equivalent to keeping constant the initially purchased quantities: no rebalancing trades are made.

\subsection{Performance Metrics Analysis}

In this section we discuss the metrics used to assess portfolio performance. As explained previously, our methodology yields various portfolio types, characterized by their restrictions on the maximum and minimum weights, the maximum amount of leverage, and other criteria.

We apply a set of tests to compare the performance of each portfolio with that of the market portfolio. The metrics used in these comparisons are based on De Miguel, Garlappi and Uppal (2007) [10] and explained below.

\subsubsection{Sharpe Ratio Test}

We begin by computing the Sharpe ratio of each of the portfolios. For each $i \in\{1,2, \cdots, N\}$ we have:

$$
\widehat{S R}=\frac{\widehat{\mu_{t}}}{\widehat{\sigma_{t}}}
$$

where $\widehat{\mu}_{t}, \widehat{\sigma}_{t}$ and $\widehat{S R}_{i}$ are respectively the mean return, the standard devia- 
tion, and the Sharpe ratio of portfolio $i$.

We then use an approach suggested by Jobson and Korkie (1981) [11] and amended by Memmel (2003) [12] to test whether the differences among the Sharpe ratios are significant. Let

$$
H_{0}: \widehat{S R}_{j}-\widehat{S R}_{M}=0
$$

where $j \in\{1,2, \cdots, N\} / M$ and $M$ is the market portfolio. We then compute the $\hat{z}_{i M}$ statistic, whose asymptotic distribution is the standard normal distribution ${ }^{4}$ :

$$
\hat{z}_{i M}=\frac{\hat{\sigma}_{i} \hat{\mu}_{i}-\hat{\sigma}_{M} \hat{\mu}_{M}}{\sqrt{\hat{v}}}
$$

such that

$$
\hat{v}=\frac{1}{T}\left(2 \hat{\sigma}_{i}^{2} \hat{\sigma}_{M}^{2}-2 \hat{\sigma}_{i} \hat{\sigma}_{M} \hat{\sigma}_{I M}+\frac{1}{2} \hat{\mu}_{i}^{2} \hat{\sigma}_{M}^{2}+\frac{1}{2} \hat{\mu}_{M}^{2} \hat{\sigma}_{i}^{2}-\frac{\hat{\mu}_{i} \hat{\mu}_{M}}{\hat{\sigma}_{i} \hat{\sigma}_{M}} \sigma_{i M}^{2}\right)
$$

Therefore, just as in a $t$ test, we need only compare the value obtained above with the critical value given by the normal distribution: if the critical value is lower than the computed value then we reject the null hypothesis that the Sharpe ratios of the two portfolios are equal.

\subsubsection{Certainty Equivalence Analysis}

The certainty equivalent test goes largely as above. It also consists in calculating a performance metric for all portfolios followed by a difference test between the assembled portfolios and the market portfolio. In this new case the metric is the certainty equivalent ${ }^{3}$ of the portfolio, obtained as follows:

$$
E Q=\hat{\mu}_{i}-\frac{\gamma}{2} \hat{\sigma}_{i}^{2}
$$

where $i \in\{1,2, \cdots, N\}$ and $\gamma$ is the risk aversion parameter.

Having first computed the certainty equivalent for all portfolios using (20), we perform a difference test on the values so obtained.

Specifically, letting $f_{i M}(v)=\left[\mu_{i}-\frac{\gamma}{2} \sigma_{i}^{2}\right]-\left[\mu_{M}-\frac{\gamma}{2} \sigma_{M}^{2}\right]$, we test the null hypothesis $H_{0}: f(v)=0$, knowing that:

$$
\hat{\Psi}_{i M}=\sqrt{T}\left(f_{i M}(\hat{v})-f_{i M}(v)\right) \sim N\left(0, \frac{\partial f^{\mathrm{T}}}{\partial v} \Theta \frac{\partial f}{\partial v}\right)
$$

where

$$
\Theta=\left[\begin{array}{rlrl}
\sigma_{i}^{2} & \sigma_{i M} & 0 & 0 \\
\sigma_{M i} & \sigma_{M}^{2} & 0 & 0 \\
0 & 0 & 2 \sigma_{i}^{4} & 2 \sigma_{i M}^{2} \\
0 & 0 & 2 \sigma_{M i}^{2} & 2 \sigma_{M}^{4}
\end{array}\right]
$$

${ }^{3}$ The certainty equivalent is the increase in the risk-free rate that would render the agent indifferent between the risky investment and the risk-free investment. As was pointed out by De Miguel, Garlappi and Uppal (2007) [10], Equation (20) corresponds not to the certainty equivalent but instead to the expected utility of a mean-variance investor, which is in turn approximated by the certainty equivalent of a quadratic-utility investor. 
We analyse the results as before: if the computed value is higher than the critical value then we reject the null hypothesis. Note that we are setting the risk aversion parameter $\gamma$ equal to 1 .

\section{Results}

We now discuss the performance of the portfolios. The analysis is organized as follows: we first compare the returns of the alpha portfolios with the return of the market portfolio over the whole period; we then compare the alpha and market portfolios by using the performance metrics described in section 4.2.

The various types of portfolios involved in this paper are summarized in Table 1, which specifies for each portfolio the weighting convention (equal weight or value weight), the abbreviated names, and some explanatory remarks.

The first portfolio is the market portfolio, whose performance is used as the benchmark. The portfolios are classified into four types, each containing two portfolios: one with equal weights and one with value weights as explained in section 4.1.5.

The first of the four types are the Markowitz long-only portfolios, which consist of portfolios without any short positions, as explained in section 4.1.2.3. Second, the naive portfolios follow the simpler procedure described in section 4.1.2.1. Third, there are the (restricted-weight) Markowitz portfolios whose methodology has been described in section 4.1.2.2 and whose weights are restricted to a specific range, displayed in Table 1 as going from $-X$ to $Y$. Finally, the bounded leverage portfolios, which were described in section 4.1.2.4, have leverage limited to 10 .

\subsection{Alpha Portfolio Analysis}

We now compare the returns of the alpha and market portfolios. Table 2 displays the cumulative average returns for each portfolio and the market portfolio

Table 1. Analysed portfolios.

\begin{tabular}{cccc}
\hline Type & Weight & Abbreviation & Remark \\
\hline Market & - & Mrkt & - \\
Markowitz long-only & Equal & m-long. $E$ & - \\
& Value & m-long. $V$ & Maximum leverage equal to 10 \\
Markowitz leverage & Equal & m-lvrg. $E$ & \\
& Value & $m$-lvrg. $V$ & \\
Naïve & Equal & naive.E & \\
& Value & naive. $V$ & \\
& Equal & $m$-X.Y.E & Weights must be between $-X$ and $+Y$ \\
Markowitz & Value & $m$-X.Y.V & \\
&
\end{tabular}

The first column displays the name of the portfolio, the second column indicates whether the portfolio was assembled with constant weights (Equal) or weights varying according to past performance (Value), and the third column includes some explanatory remarks. 
Table 2. Mean return rates.

\begin{tabular}{|c|c|c|c|c|c|c|c|}
\hline \multicolumn{8}{|c|}{ Mean returns (\% per day) } \\
\hline \multirow{3}{*}{ Portfolios } & \multicolumn{6}{|c|}{ Period } & \multirow{3}{*}{ Full period } \\
\hline & 2002 & 2004 & 2006 & 2008 & 2010 & 2012 & \\
\hline & 2003 & 2005 & 2007 & 2009 & 2011 & 2013 & \\
\hline Mrkt & 0.05 & 0.04 & 0.08 & -0.03 & -0.04 & -0.01 & 0.01 \\
\hline$m$-long. $E$ & 0.06 & -0.02 & -0.07 & 0.12 & 0.06 & 0.03 & 0.03 \\
\hline$m$-long. $V$ & 0.06 & -0.02 & -0.08 & 0.15 & 0.05 & 0.05 & 0.03 \\
\hline$m$-lvrg. $E$ & 0.24 & 0.03 & 0.04 & -0.03 & 0.21 & 0.23 & 0.12 \\
\hline$m$-lvrg. $V$ & 0.22 & 0.03 & 0.01 & 0.04 & 0.19 & 0.23 & 0.12 \\
\hline naive. $E$ & 0.15 & -0.01 & 0.01 & -0.04 & 0.11 & 0.08 & 0.05 \\
\hline naive. $V$ & 0.14 & -0.02 & 0.00 & -0.04 & 0.10 & 0.07 & 0.04 \\
\hline$m-10.10 . E$ & 0.14 & -0.02 & 0.05 & -0.01 & 0.22 & 0.19 & 0.10 \\
\hline$m-10.10 . V$ & 0.12 & -0.02 & 0.05 & -0.02 & 0.2 & 0.19 & 0.08 \\
\hline$m-10.30 . E$ & 0.25 & 0.03 & 0.04 & -0.01 & 0.24 & 0.25 & 0.13 \\
\hline$m-10.30 . V$ & 0.23 & 0.03 & 0.02 & 0.00 & 0.22 & 0.25 & 0.13 \\
\hline$m-20.20 . E$ & 0.24 & 0.02 & 0.09 & 0.02 & 0.21 & 0.23 & 0.14 \\
\hline$m-20.20 . V$ & 0.22 & 0.02 & 0.07 & 0.03 & 0.19 & 0.25 & 0.12 \\
\hline$m-20.30 . E$ & 0.26 & 0.03 & 0.10 & -0.02 & 0.23 & 0.23 & 0.13 \\
\hline$m-20.30 . V$ & 0.24 & 0.03 & 0.08 & -0.03 & 0.21 & 0.24 & 0.12 \\
\hline
\end{tabular}

Average per-day (\% p.d.) rates of cumulative returns of the alpha and market portfolios. The rates are grouped into two-year intervals. The "Full period" column displays the average per-day rates over the entire period.

in terms of daily return (\% p.d.) and the values were grouped into two-year intervals.

Note that the returns of the alpha portfolios are often much higher than those of the market portfolio. This is not true, however, between 2004 and 2009, when alpha returns generally tie with or are lower than the market returns.

The results indicate a partial success in assembling portfolios that beat the market. Though the alpha returns are higher over the whole period, this does not occur consistently over time: there are fairly long periods during which the strategies do not accurately predict the asset alphas.

It follows that inertial behavior of the asset alphas-which was used as a hypothesis in this work-cannot be fully confirmed. On the other hand, this inertia did generate significant gains when the entire period is considered. As we shall see shortly, the results do show that the resulting gains justify the additional risk incurred. We now check this using the metrics which were discussed in sections 2.3 and 2.4 .

\subsection{Sharpe Ratio Analysis}

We now turn our attention to comparing the Sharpe ratio of the portfolios. The results are summed up in Table 3. The first column names the portfolios, the 
Table 3. Sharpe ratio difference tests.

\begin{tabular}{|c|c|c|c|}
\hline Portfolio & Sharpe ratio $\hat{\mu}_{i} / \hat{\sigma}_{i}$ & Statistic $\hat{z}_{i M} \sim N(0,1)$ & $\mathrm{p}$-value \\
\hline Mrkt & 0.015 & - & - \\
\hline$m$-long. $E$ & 0.028 & 0.47 & 0.638 \\
\hline$m$-long. $V$ & 0.031 & 0.58 & 0.562 \\
\hline$m$-lvrg. $E$ & $0.059^{*}$ & 1.66 & 0.097 \\
\hline$m$-lvrg. $V$ & $0.059^{*}$ & 1.66 & 0.098 \\
\hline naive. $E$ & 0.049 & 1.31 & 0.191 \\
\hline naive. $V$ & 0.045 & 1.13 & 0.257 \\
\hline$m-10.10 . E$ & 0.057 & 1.58 & 0.114 \\
\hline$m-10.10 . V$ & 0.051 & 1.37 & 0.172 \\
\hline$m-10.30 . E$ & $0.061^{*}$ & 1.74 & 0.082 \\
\hline$m-10.30 . V$ & $0.059^{*}$ & 1.67 & 0.095 \\
\hline$m-20.20 . E$ & 0.057 & 1.60 & 0.110 \\
\hline$m-20.20 . V$ & 0.056 & 1.55 & 0.121 \\
\hline$m-20.30 . E$ & 0.055 & 1.54 & 0.124 \\
\hline$m-20.30 . V$ & 0.052 & 1.41 & 0.157 \\
\hline
\end{tabular}

Given each portfolio we tested the hypothesis $H_{0}: \hat{\mu}_{i} / \hat{\sigma}_{i}-\hat{\mu}_{M} / \hat{\sigma}_{M}=0$, where $\mu$ is the mean portfolio return, $\sigma$ is its standard deviation, $i$ is the index of the alpha portfolio and $M$ is the market portfolio. Significance levels: ${ }^{* *} 1 \%,{ }^{*} 5 \%,{ }^{\star} 10 \%$

second column lists their Sharpe ratios, and the third column shows the $\hat{z}_{i M}$ statistic regarding the difference test between the Sharpe ratios of the alpha and market portfolios that was discussed in section 4.2.1. Finally, the last column reports the test $\mathrm{p}$-value.

As shown in the Table, the market portfolio had a Sharpe ratio of 0.015 , the lowest among all analysed portfolios. Here are the remaining portfolios in decreasing order of performance as measured by the Sharpe ratio: $m$-10.30.E, $m$-10.30. $V, m$-lvrg. $E, m$-lvrg. $V m$-10.10.E, $m$-20.20.E, $m$-20.20. $V, m$-20.30.E, $m$-20.30. $V, m$-10.10. $V$, naive. $E$, naive. $V$, $m$-long. $V$ and $m$-long. $E$. Their Sharpe ratios ranged from 0.028 to 0.061 .

Although all of the alpha portfolios have higher Sharpe ratios than the market portfolio, only the top four were significantly higher, all four at the $10 \%$ significance level. Note also that there was little difference in performance between the equal-weighted and value-weighted portfolios. As one can see the performance was essentially the same regardless of weight used.

\subsection{Certainty Equivalent Analysis}

We now analyse the alpha portfolios via the certainty equivalent differences tests discussed in section 4.2.2. As previously explained, these tests compare the certainty equivalent of each alpha portfolio with the market portfolio. We set out the results in Table 4, whose columns display the following information from left to right: abbreviated portfolio name, value of the certainty equivalent, value of the $\Psi_{i M}$ statistic, and the test p-value. 
Table 4. Certainty equivalent difference tests.

\begin{tabular}{|c|c|c|c|}
\hline Portfolio & Certainty equivalent $\hat{\mu}_{K}-\frac{1}{2} \hat{\sigma}_{K}^{2}$ & Statistic $\Psi_{i M} \sim N(0,1)$ & $p$-value \\
\hline Mrkt & 0.00018 & - & - \\
\hline$m$-long. $E$ & 0.00033 & 0.388 & 0.698 \\
\hline$m$-long. $V$ & 0.00038 & 0.495 & 0.621 \\
\hline$m$-lvrg. $E$ & $0.00128^{\star *}$ & 2.040 & 0.041 \\
\hline$m$-lvrg. $V$ & $0.00127^{\star *}$ & 2.034 & 0.042 \\
\hline naive. $E$ & 0.00049 & 0.876 & 0.381 \\
\hline naive. $V$ & 0.00044 & 0.745 & 0.456 \\
\hline$m-10.10 . E$ & $0.00099^{*}$ & 1.756 & 0.079 \\
\hline$m-10.10 . V$ & 0.00086 & 1.511 & 0.131 \\
\hline$m-10.30 . E$ & $0.00140^{* *}$ & 2.178 & 0.029 \\
\hline$m-10.30 . V$ & $0.00132^{\star \star}$ & 2.082 & 0.037 \\
\hline$m-20.20 . E$ & $0.00144^{\star *}$ & 2.064 & 0.039 \\
\hline$m-20.20 . V$ & $0.00137^{\star *}$ & 1.995 & 0.046 \\
\hline$m-20.30 . E$ & $0.00152^{\star *}$ & 2.029 & 0.042 \\
\hline$m-20.30 . V$ & $0.00138^{*}$ & 1.861 & 0.063 \\
\hline
\end{tabular}

Given each portfolio we tested the hypothesis $H_{0}: \hat{\Psi}_{i M}=\sqrt{T}\left(f_{i M}(\hat{v})-f_{i M}(v)\right)=0$, where

$f_{i M}(v)=\left[\mu_{i}-\frac{\gamma}{2} \sigma_{i}^{2}\right]-\left[\mu_{M}-\frac{\gamma}{2} \sigma_{M}^{2}\right], \mu$ is the mean portfolio return, $\sigma$ is the standard deviation, $i$ is the index of the alpha portfolio, $M$ is the market portfolio, and $\gamma$ is the risk aversion parameter, set at $\gamma=1$. Significance levels: ${ }^{* * *} 1 \%,{ }^{* *} 5 \%,{ }^{*} 10 \%$.

As in the previous test, the market portfolio had the worst result among all portfolios. The values for the alpha portfolios, however, are not always statistically higher than the market portfolio.

Here are the remaining portfolios in decreasing order of performance as measured by the certainty equivalent: $m$-20.30.E, $m-20.20 . E, m-10.30 . E$, $m$-20.30. $V, m-20.20 . V, m-10: 30 . V, m$-lvrg. $E, m$-lvrg. $V, m-10.10 . E, m-10.10 . V$, naive. $E$, naive. $V, m$-long. $V$ and $m$-long. $E$. Nine of the top alpha portfolios values have certainty equivalents statistically above the market portfolio at a $10 \%$ significance level.

The certainty equivalent test results are in general very similar to the Sharpe ratio test results. The lists of portfolios ordered by decreasing performance are similar for the different metrics used. There is moreover little difference in performance, again as measured by the various metrics, between equally-weighted and value-weighted portfolios of otherwise the same types.

\section{Conclusions}

We evaluated an investment strategy by betting on pricing errors made by the CAPM model, under the hypothesis that these errors are non-zero and persist over time. In order to do so, we first computed for each asset its expected pricing 
error (the asset alpha), defined as the expected return when one takes a long position in the asset while shorting the market with weight equivalent to the asset risk (beta). We essentially built optimal Markowitz (1952) [1] portfolios, replacing the expected returns vector by the expected error vector.

Our results show that over the entire period of analysis, all of the alpha portfolios obtained above-market returns. These results were not significantly affected by the choice of equal-valued or value-weighted portfolios. Except over some shorter periods, our hypothesis was fully confirmed, that is, the performance of our alpha portfolios was significantly better than that of the market portfolio. Furthermore, two metrics were used to assess portfolio performance, the Sharpe ratio and the certainty equivalent. Both metrics confirmed that the performances of the alpha portfolios were better than that was observed for the market portfolio. In other words, the pricing error of the CAPM model has an inertial component that allows one to obtain returns that are significantly above market returns.

\section{References}

[1] Markowitz, H. (1952) Portfolio Selection. The Journal of Finance, 7, 77-91. https://doi.org/10.1111/j.1540-6261.1952.tb01525.x

[2] Sharpe, W.F. (1964) Capital Asset Prices: A Theory of Market Equilibrium under Conditions of Risk. The Journal of Finance, 19, 425-442. https://doi.org/10.1111/j.1540-6261.1964.tb02865.x

[3] Lintner, J. (1965) The Valuation of Risk Assets and the Selection of Risky Investments in Stock Portfolios and Capital Budgets. The Review of Economics and Statistics, 17, 13-37. https://doi.org/10.2307/1924119

[4] Fama, E.F. and French, K.R. (2004) The Capital Asset Pricing Model: Theory and Evidence. Journal of Economic Perspectives, 18, 25-46. https://doi.org/10.1257/0895330042162430

[5] Banz, R.W. (1981) The Relationship between Return and Market Value of Common Stocks. Journal of Financial Economics, 9, 3-18. https://doi.org/10.1016/0304-405X(81)90018-0

[6] Rosenberg, B., Reid, K. and Lanstein, R. (1985) Persuasive Evidence of Market Inefficiency. The Journal of Portfolio Management, Institutional Investor Journals, 11, 9-16.

[7] Fama, E.F. and French, K.R. (1993) Common Risk Factors in the Returns on Stocks and Bonds. Journal of Financial Economics, 33, 3-56. https://doi.org/10.1016/0304-405X(93)90023-5

[8] Jegadeesh, N. and Titman, S. (1993) Returns to Buying Winners and Selling Losers: Implications for Stock Market Efficiency. The Journal of Finance, 48, 65. https://doi.org/10.1111/j.1540-6261.1993.tb04702.x

[9] Fama, E.F. and French, K.R. (2014) A Five-Factor Asset Pricing Model. Journal of Financial Economics, 116, 1-22. https://doi.org/10.1016/j.jfineco.2014.10.010

[10] Demiguel, V., et al. (2007) Optimal versus Naive Diversification: How Inefficient Is the 1/N Portfolio Strategy? The Review of Financial Studies, 22, 3411-3447.

[11] Jobson, J.D. and Korkie, B.M. (1981) Performance Hypothesis Testing with the Sharpe and Treynor Measures. The Journal of Finance, 36, 4.

https://doi.org/10.1111/j.1540-6261.1981.tb04891.x 
[12] Memmel, C. (2003) Performance Hypothesis Testing with the Sharpe Ratio. Finance Letters, 1, 21-23.

Submit or recommend next manuscript to SCIRP and we will provide best service for you:

Accepting pre-submission inquiries through Email, Facebook, LinkedIn, Twitter, etc. A wide selection of journals (inclusive of 9 subjects, more than 200 journals)

Providing 24-hour high-quality service

User-friendly online submission system

Fair and swift peer-review system

Efficient typesetting and proofreading procedure

Display of the result of downloads and visits, as well as the number of cited articles Maximum dissemination of your research work

Submit your manuscript at: http://papersubmission.scirp.org/

Or contact ti@scirp.org 